live affected male being born, after parents proceeded with the pregnancy.

Conclusion Rigorous and expansive approaches to cascadescreening/counselling may account for decreases in preventable cases. Age of diagnosis has fallen, providing potential for more timely intervention, aided by newer diagnostic techniques that allow more accurate proband genotyping. Prenatal testing identified small numbers of affected males, facilitating parental decision-making in these select cases.

\section{RESISTANCE EXERCISES WITH BLOOD FLOW RESTRICTION IN PATIENTS WITH SPORADIC INCLUSION BODY MYOSITIS}

${ }^{1}$ Christina Liang*, 'Melanie Burk, ${ }^{1}$ Abby Wall, ${ }^{2}$ Ryan Davis, 'Libby Augustine, 'Sarah Bright, ${ }^{1}$ Whitney Brown, ${ }^{1}$ Anneliese Harris, ${ }^{1}$ Sarah Holmes, 'Sayanthinie Jeyalingam, 'Sarah Large, ${ }^{1}$ Sylvia Mai, ${ }^{1}$ Eleanor Raper, ${ }^{1}$ Hang Wah Judtih Wong, ${ }^{1}$ Pok Man Wong, ${ }^{1,2}$ Carolyn M Sue, ${ }^{1}$ Barbara Lucas. ${ }^{1}$ Royal North Shore Hospital, St Leonards, NSW, Australia; ${ }^{2}$ Kolling Institute of Medical Research, St Leonards, NSW, Australia

\subsection{6/jnnp-2019-anzan.23}

Introduction Sporadic inclusion body myositis (sIBM) is the most common muscle disease affecting older adults with no disease-modifying treatment. Resistance exercises increase muscle hypertrophy, but weakness prevents exercising with higher resistance. In healthy subjects, augmentation of lightload training with blood flow restriction improved muscle strength $^{1}$; and similar exercises were safe in the elderly. ${ }^{2}$ We therefore investigate whether resistance exercises with blood flow restriction is safe and helpful in sIBM patients. We explored methods for exercising weak leg muscles, and options for better outcome measures.

Methods A matched-control pilot study, with 12-week treatment and 4-week follow-up periods, where participants concentrated on lower limbs resistance exercises with 50\% blood flow restriction 3 times/week, at 20\%-30\% of their repetition maximum. Patients are reviewed 4-weekly for muscle strength, blood biomarkers, 2-minute walk test (2MWT), timed up-andgo (TUG) test, minimal chair height standing ability test (MCHSAT), thigh girths, and quality of life scales.

Results 4 patients with varying abilities were on the exercise arm, and 3 acted as controls. Muscle groups with MRC score $\geq 2$ were able to be exercised. We found strength testing by hand-held dynamometer was unreliable, whereas the 2MWT, TUG test and MCHSAT showed less variability. All patients could perform their exercises at significantly increased repetitions or weights by week 4, without concerning adverse events, with trend towards continued improvement over the 4-month period.

Conclusion Resistance exercises with blood flow restriction appear safe, and may be helpful in sIBM patients, even in weakened muscles, enabling improvement in muscle strength and endurance.

\section{REFERENCES}

1. Hughes L, Paton B, Rosenblatt B, Gissane C, Patterson SD. Blood flow restriction training in clinical musculoskeletal rehabilitation: a systematic review and metaanalysis. Br J Sports 2017.

2. Vechin FC, Libardi CA, Conceicao MS, et al. Comparisons between low-intensity resistance training with blood flow restriction and high-intensity resistance training on quadriceps muscle mass and strength in elderly. J Strength Cond Res 2015.

\section{THE UTILITY OF NERVE BLOOD FLOW IN THE ASSESSMENT OF PERIPHERAL NEUROPATHY}

${ }^{1,2}$ Adeniyi Borire*, ${ }^{1}$ Tushar Issar, ${ }^{3}$ Natalie Kwai, ${ }^{4}$ Leo Visser, ${ }^{5}$ Ann Poynten, ${ }^{6}$ Neil Simon, ${ }^{7}$ Matthew Kiernan, ${ }^{1}$ Arun Krishnan. ${ }^{1}$ Prince of Wales Clinical School, UNSW, Sydney, NSW, Australia; ${ }^{2}$ Liverpool Hospital, Sydney, NSW, Australia; ${ }^{3}$ School of Medical Sciences, UNSW, Sydney, NSW, Australia; ${ }^{4}$ St Elisabeth Ziekenhuis, Tilburg, Netherlands; ${ }^{5}$ Department of Endocrinology, Prince of Wales Hospital, Sydney, NSW, Australia; ${ }^{6}$ St Vincent's Clinical School, University of New South Wales, Sydney, NSW, Australia; ${ }^{7}$ Brain and Mind Centre, Sydney, NSW, Australia

\subsection{6/jnnp-2019-anzan.24}

Introduction Animal and human studies of diabetes have shown significant alterations in nerve blood flow (NBF), which may also play a role in the development of neuropathy. However, the non-invasive assessment of NBF in human subjects has remained elusive until the recent technological advancements in ultrasound (US) technology. We undertook sonographic assessment of NBF in 75 patients with type 2 diabetes and correlated the findings with neuropathy severity scores and electrophysiological parameters.

Methods Blinded median and tibial nerve ultrasound scans were performed at non-entrapment sites using a high-resolution linear probe. NBF was quantified using power Doppler techniques to obtain the vessel score (VSc) and maximum perfusion intensity (MPI). Routine nerve conduction studies were performed, and neuropathy severity was assessed using the total neuropathy score (TNS). Aged- and gender-matched controls were enrolled.

Results Diabetic nerves had higher rates of NBF detection $(28 \%)$ compared to the control group $(p<0.0001)$. Significant correlations were found between NBF parameters and nerve size $(p<0.001)$, reported sensory symptoms $(p<0.05)$ and neuropathy severity scores $(\mathrm{p}<0.001)$. The cohort with diabetes had significantly larger median $\left(8.5 \pm 0.3 \mathrm{~mm}^{2}\right.$ vs. $7.2 \pm 0.1$ $\left.\mathrm{mm}^{2}, \mathrm{p}<0.05\right)$ and tibial $\left(18.0 \pm 0.9 \mathrm{~mm}^{2}\right.$ vs. $12.8 \pm 0.5$ $\left.\mathrm{mm}^{2}, \mathrm{p}<0.05\right)$ nerves compared to controls.

Conclusions Peripheral nerve hypervascularity is detectable by US in moderate to severe diabetic neuropathy with prominent sensory dysfunction. Consistent with previous studies, the sonographic detection of NBF is a pathological finding.

\section{VARIATION IN THE ANATOMY OF THE NORMAL HUMAN OPTIC CHIASM: AN MRI STUDY}

${ }^{1,2}$ Christian J Lueck* ${ }^{2}$ Nicholas Bosler, ${ }^{3}$ Andrew Neely, ${ }^{4}$ David Ashton. ${ }^{1}$ Department of Neurology, The Canberra Hospital, Canberra, ACT, Australia; ${ }^{2}$ Medical School, Australian National University, Canberra, ACT, Australia; ${ }^{3}$ School of Engineering, University of New South Wales, Canberra, ACT, Australia; ${ }^{4}$ Department of Radiology, Canberra Hospital, Canberra, ACT, Australia

\subsection{6/jnnp-2019-anzan.25}

Introduction Compression of the optic chiasm gives rise to bitemporal hemianopia. The reason for this is unclear, but one hypothesis suggests it relates to the fact that nasal retinal fibres cross each other while temporal fibres do not. This 'crossing' hypothesis has been investigated using finite element modelling but this requires accurate anatomical data. The precise shape of the chiasm is not clear: nasal fibres may not decussate centrally (as if the chiasm were ' $\mathrm{X}$ '-shaped) but, instead, decussate paracentrally and run 